\title{
With your genes? Take one of these, three times a day
}

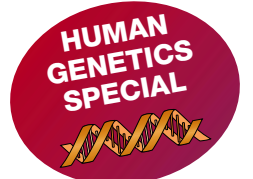

\author{
Truly 'personalized' medicine remains \\ a distant goal. But researchers are \\ now thinking about how to use
}

genomic data to avoid prescribing drugs that may kill, or won't work. Alison Abbott reports.

t causes at least 100,000 deaths each year in the United States, and is responsible for more than $10 \%$ of hospital admissions in some European countries. But this isn't some terrifying emerging infection, it's the annual toll inflicted by adverse reactions to prescription drugs. What's more, millions of people are being treated with drugs that, for them, will never do much good. Betablockers, given to reduce blood pressure, are ineffective in one-third of patients; many antidepressants don't work in half of the people who take them.

The blame lies largely with our genes, which help to determine the way in which we react to drugs. Small genetic variations between people — or polymorphisms - can alter the behaviour of proteins that carry a drug to its target cells or tissues, cripple the enzymes that activate a drug or aid its removal from the body, or alter the structure of the receptor to which a drug is supposed to bind. Variation in immune-system genes can also influence how particular drugs are tolerated. Together, these subtle genetic variations mean that the dose at which a drug will work may vary hugely from person to person. And with today's 'one-size-fits-all' prescribing, that can lead to life-threatening adverse reactions or to a drug completely failing to do its job.

Yet the genomics revolution has given us the tools to identify people who don't fit the standard prescribing mould. Single nucleotide polymorphisms, or SNPs, are single-letter changes in the genetic code that are scattered throughout the genome. They can now be profiled with increasing efficiency, and used to highlight polymorphic genes that influence our response to individual drugs. Will we see a seismic shift in prescribing practice? With barely a handful of concrete examples to go on so far, it's too early to judge the true promise of personalized medicine - but the pharmaceutical ground is certainly starting to shake.

Regulatory agencies such as the US Food and Drug Administration (FDA) are starting to consider whether or not some drugs should be labelled as being suitable only for individuals with a defined genetic profile. Next month in Washington DC, the FDA will hold a workshop on the topic. Drug companies are preparing themselves for a new lie of the land. Already, three-quarters of the clinical trials they submit to the FDA for approval include provision for sampling and storing blood for any genetic analysis that may be required in the future. But it is academic researchers who are most enthusiastically pushing things forward.

Pharmacogenetics - the study of the influence of genetic variation on drug responses — isn't a new discipline. But until the advent of SNP analysis, progress was laborious. An early example of the field's promise came with the introduction, in the 1950 s, of the muscle relaxant succinyl-
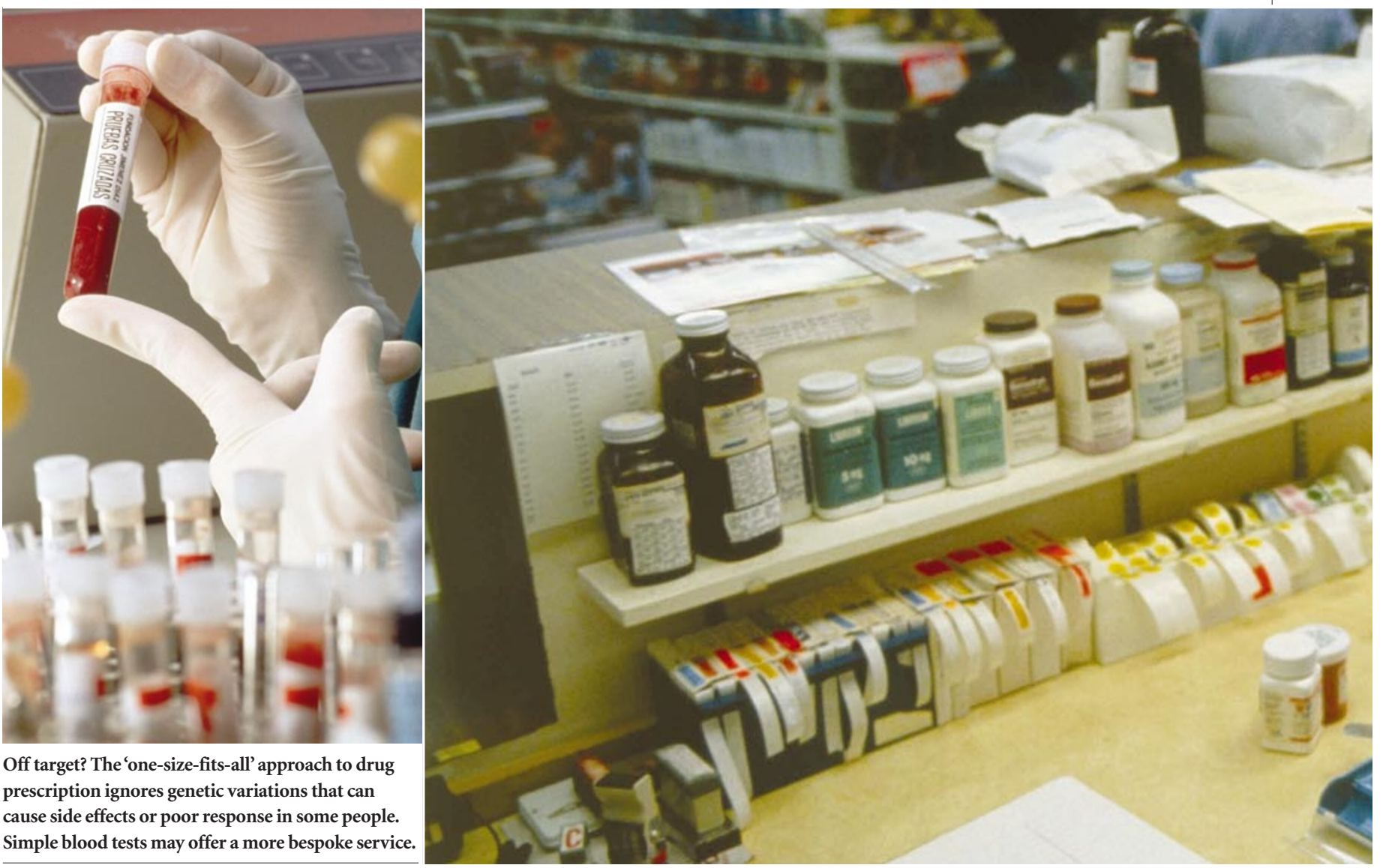

Off target? The 'one-size-fits-all' approach to drug prescription ignores genetic variations that can cause side effects or poor response in some people. Simple blood tests may offer a more bespoke service. 
choline. To their horror, anaesthetists found that a small proportion of patients given the drug went into life-threatening respiratory arrest on the operating table. Geneticists were able to show why. Succinylcholine is normally metabolized very efficiently by an enzyme called cholinesterase. But 1 in 2,500 people carry two defective copies of the gene for this enzyme, and suffer deep and prolonged paralysis of muscles, including those needed for breathing, when given the drug.

\section{Hidden from view}

Being rare, such reactions are unlikely to be noticed in clinical trials, which typically involve a couple of thousand patients. They tend to be revealed when a drug is widely prescribed - which is why regulatory agencies require drug companies to supply information about adverse drug reactions for years after approval. If such reactions occur in an identifiable group of patients, the drug's labelling can be modified to exclude them. In the case of succinylcholine, bad responders are identified by measuring cholinesterase activity in the blood.

The FDA is now close to making a landmark decision to relabel an approved drug to exclude bad responders identified by SNP analysis alone. Mercaptopurine, approved by the agency in the 1950s, is used to treat childhood leukaemias and other cancers. Like all anticancer agents, it is intrinsically toxic, and

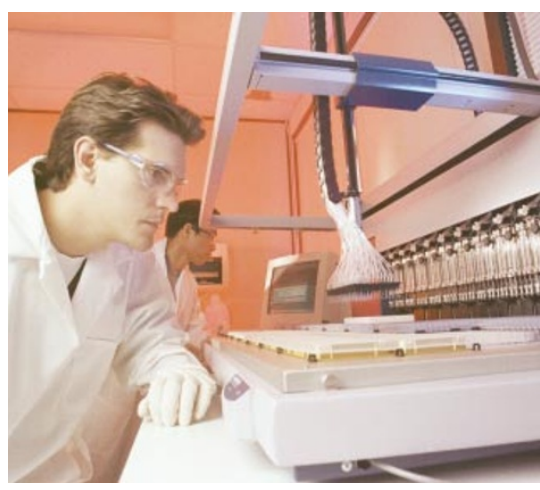

Analytical express: genotyping of samples has become a highly automated business.

there is a narrow dose range within which the therapeutic benefit outweighs its toxicity. But some patients suffer life-threatening bonemarrow damage at doses tolerated well by others. William Evans, scientific director of St Jude Children's Research Hospital in Memphis, Tennessee, suspected that this could be linked to polymorphism in the gene for the enzyme thiopurine methyltransferase, which metabolizes the drug.

In the mid-1990s, Evans began to compare the sequences of this gene in patients with and without the toxic reaction. He identified three SNPs, any of which resulted in an enzyme that allowed mercaptopurine to linger in the body at dangerous levels ${ }^{1}$.

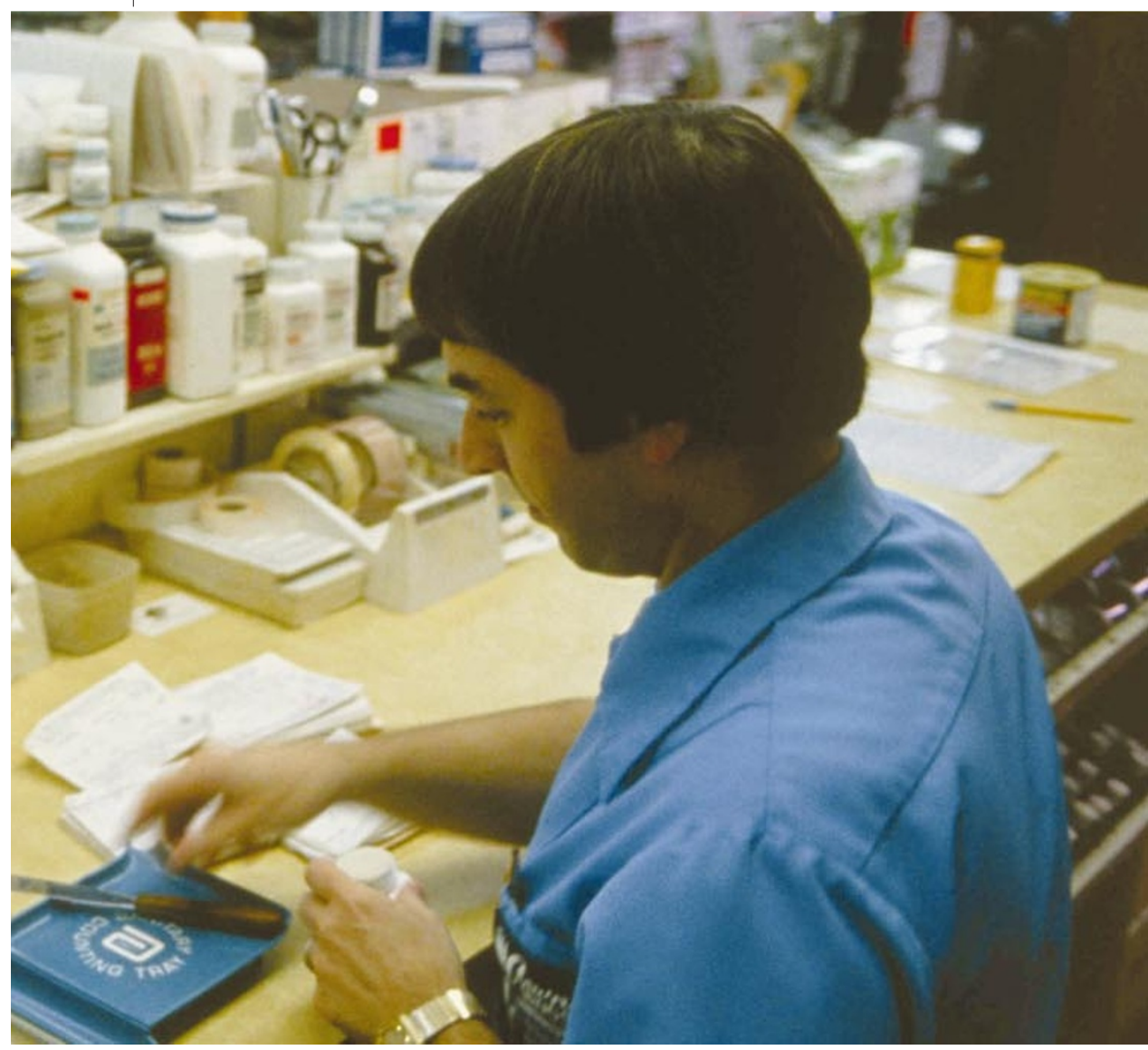

"Altogether we found that $70 \%$ of cases of toxicity have carried one or more mutations," says Evans. "SNP testing gives clinicians a much more robust and easy-to-handle method of estimating this risk than measuring activity of the enzyme." Even before the FDA has given its seal of approval, some hospitals are now subjecting patients to genetic tests before prescribing mercaptopurine.

Most research in this field has similarly focused on polymorphisms affecting drug metabolism. Pharmacogeneticist Magnus Ingelman-Sundberg of the Karolinska Institute in Stockholm, Sweden, estimates that the efficacy or safety of $20 \%$ of the drugs now on the market is associated with a polymorphism in a single gene for a metabolizing enzyme. A similar proportion may be influenced by polymorphisms in multiple drug-metabolizing genes. Factor in the largely unknown quantity of polymorphisms influencing the transport of drugs or their interaction with receptors, plus immune-system genes that influence tolerance to drugs, and it's clear that pharmacogeneticists have barely scratched the surface.

\section{Family fortunes}

One key group of drug-metabolizing enzymes is the P450 family. These enzymes are produced in the liver and oxidize foreign chemicals. Of the 57 genes for P450 enzymes that have been identified in humans, three CYP2D6, CYP2C9 and CYP3A4 - are particularly important for drug metabolism. CYP3A4 metabolizes half of all prescribed drugs but seems to have few polymorphisms that could affect drug function; the polymorphic CYP2D6 is involved in the metabolism of a quarter of prescribed drugs — including beta-blockers and antidepressants; CYP2C9 is involved in the metabolism of $5 \%$ of drugs and is also highly polymorphic ${ }^{2}$.

Although many of these polymorphisms will have no effect on enzyme function, some have already been linked to the failure, in certain patients, of commonly prescribed drugs. For example, the painkiller codeine depends for its effect on being oxidized to morphine by CYP2D6; people with a particular polymorphism - up to $10 \%$ of the population don't get pain relief. The hit-and-miss efficacy of antidepressants such as Prozac may also be partly the result of CYP2D6 polymorphisms ${ }^{3}$.

Other P450 polymorphisms are associated with serious adverse drug reactions. For example, the widely used anticoagulant warfarin is metabolized by the enzyme encoded by CYP2C9, and the small proportion of patients with polymorphisms that reduce the enzyme's activity can suffer life-threatening bleeding unless warfarin doses are reduced ${ }^{4}$. In one dramatic case in the 1980s, a drug for angina called perhexiline had to be pulled off the market when it turned out to be toxic to liver and nerve cells in rare cases of a particular CYP2D6 polymorphism. Ingelman-Sundberg estimates that some $80 \%$ of the most 
serious adverse drug reactions involve drugs metabolized by polymorphic P450 enzymes. "We can learn a lot about how to prescribe to patients by thinking about an individual's drug-metabolizing enzyme profile," he says.

With this in mind, several genomics companies are manufacturing DNA microarrays to identify common SNPs that influence the activity of P450 enzymes, and some hospitals are starting to use them. In the long run, such chips could help not just to avoid dangerous reactions to drugs, but also in deciding appropriate drug doses, and selecting which drug cocktails to give to patients with complex conditions such as cardiovascular disease.

\section{Profiles on parade}

Given the promise of pharmacogenetics, funding agencies are starting to invest. In June, the UK Department of Health ear-

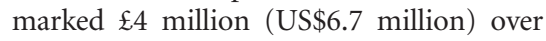
three years for pharmacogenetic research into existing drugs, and in 2001 the US National Institutes of Health (NIH) established a Pharmacogenetics Research Network through which 12 partners across the country, including Evans, will share their experiences and post results of large studies on a public website, to encourage further research. These studies include the investigation of polymorphisms in neurotransmitter receptors relevant to antidepressant therapy, and those involved in cell-signalling pathways that help to regulate blood pressure.

Another member of the NIH pharmacogenetics network is Jeffrey Drazen of Harvard Medical School in Boston, editor-in-chief of The New England Journal of Medicine. An increasing number of papers submitted to the journal are relating treatment outcomes to particular genetic profiles, but Drazen notes that academic studies tend to be small, which limits their statistical power.

Larger studies will be needed to determine the influence of groups of SNPs on drug responses in diseases, such as cardiovascular and psychiatric conditions, which are influenced by multiple genes. This will probably involve searching for informative SNPs across the entire genome - an expensive process that would mark the transition from conventional pharmacogenetics to the era of high-throughput phamacogenomics.

Such projects may require the financial muscle of the drug industry. But for now, most companies are reluctant to get involved: their priority is bringing lucrative new drugs to the widest possible market, rather than limiting their use to patients with particular genetic profiles. "Our general philosophy is not to initiate a drug-development programme that would limit the group of patients a drug could treat," says Brian Spear, director of pharmacogenetics at Abbott Laboratories in Illinois.

Drugs firms also worry that genomic information could be interpreted with excessive caution by regulatory officials — keeping



Allen Roses has identified genetic polymorphisms that cause severe side effects in an AIDS therapy.

useful drugs off the market. Larry Lesko, director of the FDA's office of clinical pharmacology and biopharmaceutics, is trying to address these worries. "Two or three years ago, we became aware of concern from companies that wanted to integrate pharmacogenetics into their drug-development programmes but were afraid we would overreact," he says.

Lesko created the concept of a 'safe haven' for genomic data. This allows information to be deposited so that, for instance, companies can make claims about a drug's efficacy in a defined population. Until better methods of analysing the data are developed, the FDA won't use this information to make judgements about safety. The concept is still being refined, but a proposal will be discussed at the November FDA workshop. "We hope that it will make companies feel freer to explore pharmacogenomics," says Lesko.

\section{Blood banked}

Most companies have seen the writing on the wall, and now store blood samples from clinical trials in case they need to analyse them retrospectively. But they want to see methods for pharmacogenomic analysis become more reliable before routinely making them part of their clinical trials. "We still don't know how predictive SNPs will be in complex diseases where lots of different factors may compensate for the effect of a polymorphism," says Klaus Lindpaintner, director of Roche Genetics in Basel, Switzerland. "Another, sometimes overlooked, problem is lack of good clinical data that show how SNPs relate to clinical responses."

Against this conservative backdrop, Allen Roses, senior vice-president of genetics research with GlaxoSmithKline (GSK) in Research Triangle Park, North Carolina, stands out as an enthusiast for SNP analysis. "Ten years down the road, the public will be insisting that even suppliers of vitamin supplements are required to supply information on polymorphisms," he predicts. GSK has several pharmacogenetic projects running, some of which involve scanning the whole genome for thousands or tens of thousands of
SNPs using various technologies. "This is simpler and cheaper than most people imagine," says Roses.

It will be years before all of these studies will be completed and published, but Roses has won praise from researchers for an initial foray into pharmacogenomics, published last year in The Lancet ${ }^{5}$. His team went through the GSK database of clinical trials to identify 85 patients who had taken the AIDS drug abacavir and experienced a violent immune reaction to it. By comparing hundreds of SNPs from these patients with those from others who did not suffer the lifethreatening side effect, Roses identified three SNPs associated with immune-system genes that can be used to identify patients who shouldn't be given abacavir.

\section{Trial-busters}

Most companies believe that pharmacogenomics could offer a way to increase the efficiency of clinical trials. In early-phase trials, a new drug is tested on about 250 patients to see if it causes toxicity and to see whether it works. On the basis of these results, regulatory authorities then decide whether to give the go-ahead for larger 'phase III' studies. If a particular genotype can be distinguished that is associated with a good response to a drug, then patients with such a genotype could be selected for the phase III trial. This could allow companies to recoup their investment in candidate drugs that ultimately prove to be effective only in a proportion of people. The FDA is considering this, but Lesko points out that safety studies would still have to be carried out in non-responders, which could mean expanding earlier phases of the trials.

As the potential of pharmacogenomics becomes clearer, its application by both drug companies and health services will be dictated by balancing costs and benefits. How many SNPs will be needed to make accurate predictions for each drug and condition, and how much will it cost to analyse them? Will the cost be worth it if the clinical benefit is small?

Those won't be easy questions to answer. But Drazen believes that the benefits of pharmacogenomics will be visible in the clinic within a decade. Currently, he notes, a doctor faced with a depressed patient has to choose from a long list of drugs, all of which work only in some patients, and carry a high risk of undesirable effects. "In future, that physician would send off a blood sample for analysis and get back a ranked list of best options for that individual patient," says Drazen. For patients who are subjected to the current prescribing lottery, that day can't come soon enough.

Alison Abbott is Nature's senior European correspondent.

1. Krynetski, E. Y. \& Evans, W. E. Oncogene 22, 7403-7413 (2003). 2. Pirmohamed, M. \& Park, B. K. Toxicology 192, 23-32 (2003).

3. Arranz, M. J., Mancama, D. T. \& Kerwin, R. W.

Curr. Pharmacogenomics 1, 151-158 (2003).

4. Higashi, M. K. et al. J. Am. Med. Assoc. 287, 1690-1698 (2002).

5. Hetherington, S. et al. Lancet 359, 1121-1122 (2002). 